\title{
Approximations for Weakly Nonlinear Evolution Equations
}

\author{
By Milan Miklaveið
}

\begin{abstract}
Convergence of approximations for a large class of weakly nonlinear parabolic and hyperbolic equations is proven. The main emphasis is on proving convergence of finite element and spectral Galerkin approximations of solutions to the weakly nonlinear wave equation

$$
u^{\prime \prime}(t)+A u(t)=F\left(t, u(t), u^{\prime}(t)\right), \quad u(0)=x_{0}, u^{\prime}(0)=y_{0},
$$

under minimal assumptions on the linear operator $A$ and on the approximation spaces. $A$ can be a very general elliptic operator (not just of 2 nd order and not necessarily in a bounded domain); $A$ can also be very singular and degenerate. The results apply also to systems of equations. Verification of the hypotheses is completely elementary for a large class of problems.
\end{abstract}

1. Overview. Approximations of solutions to initial value problems of the form

$$
u^{\prime}(t)+A u(t)=F(t, u), \quad u(0)=x_{0},
$$

are studied. The basic results, in rather abstract form, are presented in Section 2. They can be used to prove convergence of finite difference, finite element, spectral, etc., approximations of weakly nonlinear hyperbolic and parabolic equations. Throughout the paper it is assumed that the nonlinear operator $F$ is Lipschitz continuous in $u$, and for this reason, (1) is said to be weakly nonlinear ( $-A$ is the generator of a strongly continuous semigroup). The approach used to prove these results has origins in works of Kato [17], Trotter [29], Segal [27].

Section 3 contains a version of the results of Section 2 that is suitable for proving convergence of finite element approximations of solutions to the weakly nonlinear wave equation

$$
u^{\prime \prime}(t)+A u(t)=F\left(t, u(t), u^{\prime}(t)\right), \quad u(0)=x_{0}, u^{\prime}(0)=y_{0}
$$

(Theorem 3.5) and to weakly nonlinear parabolic problems (Theorem 3.3). Theorem 3.5 is new. The main emphasis is on requiring as little as possible on the linear operator $A$ and on the approximation spaces. $A$ can be a very general elliptic operator (not just of 2 nd order and not necessarily in a bounded domain); $A$ can also be very singular and degenerate.

Theorem 4.2 of Section 4 is perhaps the most interesting new result. It is a consequence of Theorem 3.5 and of the remarkable fact that sectorial operators have Friedrichs extensions. By using the Friedrichs extension one can bypass the "energy space" that is used in variational methods and this makes it possible to state the assumptions of Theorem 4.2 in a form that can be verified in a completely elementary way for a very general wave equation.

Received July 11, 1988.

1980 Mathematics Subject Classification (1985 Revision). Primary 65M60, 65J15, 35L70, 35L80, $47 \mathrm{~B} 44$. 
2. Basic Results. The following collection of hypotheses will be often used:

H1: $X, X_{1}, X_{2}, \ldots$ are Banach spaces; either all of them are real or all of them are complex vector spaces; all norms will be denoted by $\|\cdot\|$.

$\mathrm{H} 2: P_{n} \in \mathscr{B}\left(X, X_{n}\right), p \in[0, \infty)$ are such that $\left\|P_{n} x\right\| \leq p\|x\|$ for $x \in X, n \geq 1$.

H3: $Q_{n} \in \mathscr{B}\left(X_{n}, X\right), q \in[0, \infty)$ are such that $\left\|Q_{n} x\right\| \leq q\|x\|$ for $x \in X_{n}, n \geq 1$.

H4: $P_{n} Q_{n} x=x$ for $x \in X_{n}, n \geq 1$.

H5: $A_{n} \in \mathscr{B}\left(X_{n}\right), E_{n}(t) \equiv \exp \left(-A_{n} t\right), M \in[0, \infty), a \in \mathbf{R}$ are such that

$$
\left\|E_{n}(t)\right\| \leq M e^{-a t} \quad \text { for } t \geq 0, n \geq 1 \text {. }
$$

H6: $A$ is a densely defined linear operator in $X$.

H7: $\lambda_{0} \in(-\infty, a)$ is such that $\lambda_{0}$ is in the resolvent set of $A$, and for every $x$ in a dense subset of $X$ we have that

$$
\lim _{n \rightarrow \infty}\left\|Q_{n}\left(A_{n}-\lambda_{0}\right)^{-1} P_{n} x-\left(A-\lambda_{0}\right)^{-1} x\right\|=0 .
$$

If $Y$ and $Z$ are Banach spaces, then $\mathscr{B}(Y, Z)$ denotes the collection of all bounded linear operators with domain $Y$ and range in $Z ; \mathscr{B}(Y)=\mathscr{B}(Y, Y)$. Note that $A$ is closed by H7. The assumption that the domain of $A$ is dense can be a consequence of other assumptions [18].

Theorems similar to the following one can be found in [29], [17], [19], [10], [25], and they can be used to prove convergence of many different types of numerical approximations. In the proof of the theorem, ideas of Kato and Trotter are used [17, pp. 504-505]. The assumption that the $A_{n}$ are bounded is not needed in the following theorem; however, it is needed in all subsequent theorems. For discretization in time, see [15], [4], [17], [29], [25].

2.1. THEOREM. Assume $\mathrm{H} 1$ through $\mathrm{H} 7$. Then, $-A$ is the generator of a strongly continuous semigroup $E(t), t \geq 0$, and

(a) $\|E(t)\| \leq p q M e^{-a t}$ for $t \geq 0$;

(b) $\lim _{n \rightarrow \infty}\left\|Q_{n}\left(A_{n}-\lambda\right)^{-1} P_{n} x-(A-\lambda)^{-1} x\right\|=0$ for every $x \in X, \lambda \in(-\infty, a)$;

(c) $\lim _{n \rightarrow \infty} \sup _{t \geq 0} e^{b t}\left\|Q_{n} E_{n}(t) P_{n} x-E(t) x\right\|=0$ for every $x \in X, b \in(-\infty, a)$. 20]

Proof. Abbreviate $R_{n}(\lambda)=\left(A_{n}-\lambda\right)^{-1}, R(\lambda)=(A-\lambda)^{-1}$. H5 implies [25, p.

$$
\left\|R_{n}(\lambda)^{m}\right\| \leq M(a-\lambda)^{-m} \text { for } \lambda<a, m \geq 1, n \geq 1 .
$$

This implies that $\lim _{n \rightarrow \infty} Q_{n} R_{n}\left(\lambda_{0}\right) P_{n} x=R\left(\lambda_{0}\right) x$ for every $x$ in $X$.

If $\lambda<a, \lambda \in \rho(A)$ ( $\equiv$ resolvent set of $A$ ), then for all $n \geq 1$ we have that

$$
\begin{aligned}
& Q_{n} R_{n}(\lambda) P_{n}-R(\lambda) \\
& \quad=\left(1+\left(\lambda-\lambda_{0}\right) Q_{n} R_{n}(\lambda) P_{n}\right)\left(Q_{n} R_{n}\left(\lambda_{0}\right) P_{n}-R\left(\lambda_{0}\right)\right)\left(A-\lambda_{0}\right) R(\lambda) ;
\end{aligned}
$$

therefore,

$$
\text { if } \lambda \in(-\infty, a) \cap \rho(A) \text { then } \lim _{n \rightarrow \infty} Q_{n} R_{n}(\lambda) P_{n} x=R(\lambda) x \quad \text { for } x \in X .
$$

(2) and (1) imply that

$$
\text { if } \lambda \in(-\infty, a) \cap \rho(A) \text { then }\|R(\lambda)\| \leq p q M(a-\lambda)^{-1} .
$$


If $\mu \in(-\infty, a)$ and $\mu \notin \rho(A)$, then $\|R(\lambda)\|$ should approach $+\infty$ as $\lambda$ goes from $\lambda_{0}$ to $\mu$; however, by (3) this is not possible. Therefore, $(-\infty, a) \subset \rho(A)$ and (2) implies (b).

Induction on $m$ gives

$$
\lim _{n \rightarrow \infty}\left\|Q_{n} R_{n}(\lambda)^{m} P_{n} x-R(\lambda)^{m} x\right\|=0 \quad \text { for } x \in X, m \geq 1, \lambda<a .
$$

This and (1) imply that

$$
\left\|R(\lambda)^{m}\right\| \leq p q M(a-\lambda)^{-m} \text { for } \lambda<a, m \geq 1 .
$$

Therefore, there exists a strongly continuous semigroup $E(t), t \geq 0$, whose generator is $-A$; moreover, the bound in (a) of the theorem holds [25, p. 20].

To complete the proof, we have to show (c). This will be done in the following series of steps. Choose $\lambda<a, b<a, x \in X$. Since

$$
\frac{d}{d s} E_{n}(t-s) R_{n}(\lambda) P_{n} E(s) R(\lambda) x=E_{n}(t-s) P_{n}\left(R(\lambda)-Q_{n} R_{n}(\lambda) P_{n}\right) E(s) x
$$

we have that

$$
\begin{aligned}
& e^{b t}\left\|Q_{n} R_{n}(\lambda)\left(P_{n} E(t)-E_{n}(t) P_{n}\right) R(\lambda) x\right\| \\
& \quad=\left\|\int_{0}^{t} e^{b(t-s)} Q_{n} E_{n}(t-s) P_{n}\left(R(\lambda)-Q_{n} R_{n}(\lambda) P_{n}\right) e^{b s} E(s) x d s\right\| \\
& \quad \leq p q M \int_{0}^{\infty}\left\|\left(R(\lambda)-Q_{n} R_{n}(\lambda) P_{n}\right) e^{b s} E(s) x\right\| d s
\end{aligned}
$$

and the dominated convergence theorem (DCT) implies that

$$
\lim _{n \rightarrow \infty} \sup _{t \geq 0} e^{b t}\left\|Q_{n} R_{n}(\lambda)\left(P_{n} E(t)-E_{n}(t) P_{n}\right) R(\lambda) x\right\|=0 .
$$

Note that

$$
\begin{aligned}
e^{b t}\left(Q_{n}\right. & \left.R_{n}(\lambda) P_{n} E(t)-E(t) R(\lambda)\right) R(\lambda) x \\
= & \left(Q_{n} R_{n}(\lambda) P_{n}-R(\lambda)\right) e^{b t} E(t) R(\lambda) x \\
= & \left(Q_{n} R_{n}(\lambda) P_{n}-R(\lambda)\right) R(\lambda) x \\
& +\int_{0}^{t}\left(Q_{n} R_{n}(\lambda) P_{n}-R(\lambda)\right) e^{b s} E(s)(b-A) R(\lambda) x d s .
\end{aligned}
$$

Hence, the DCT and (4) imply that

$$
\lim _{n \rightarrow \infty} \sup _{t \geq 0} e^{b t}\left\|\left(E(t) R(\lambda)-Q_{n} R_{n}(\lambda) E_{n}(t) P_{n}\right) R(\lambda) x\right\|=0
$$

Since

$$
\begin{aligned}
& Q_{n} R_{n}(\lambda) E_{n}(t) P_{n} R(\lambda) x-Q_{n} E_{n}(t) P_{n} R(\lambda)^{2} x \\
& \quad=Q_{n} E_{n}(t) P_{n}\left(Q_{n} R_{n}(\lambda) P_{n}-R(\lambda)\right) R(\lambda) x
\end{aligned}
$$

we see that (5) implies

$$
\lim _{n \rightarrow \infty} \sup _{t \geq 0} e^{b t}\left\|\left(E(t)-Q_{n} E_{n}(t) P_{n}\right) R(\lambda)^{2} x\right\|=0 .
$$

We are done because the range of $R(\lambda)^{2}$ is equal to $\mathscr{D}\left(A^{2}\right)$, which is dense in $X$ [25, p. 6]. 
2.2. THEOREM. Let $E(t), t \geq 0$, be a strongly continuous semigroup on a Banach space $X$, and let $-A$ be its generator. Suppose $T \in(0, \infty), F:[0, T] \times X \rightarrow$ $X$ is continuous and that for some $L \in[0, \infty)$ we have that

$$
\|F(t, x)-F(t, y)\| \leq L\|x-y\| \quad \text { for } t \in[0, T], x \in X, y \in X .
$$

Then, for every $y \in X$ there exists a unique $x \in C([0, T], X)$ such that

$$
x(t)=E(t) y+\int_{0}^{t} E(t-s) F(s, x(s)) d s \quad \text { for } t \in[0, T] ;
$$

moreover, if $A \in \mathscr{B}(X)$, then this $x$ is the unique element of $C^{1}([0, T], X)$ which satisfies

$$
x(0)=y \quad \text { and } \quad x^{\prime}(t)+A x(t)=F(t, x(t)) \quad \text { for } t \in[0, T] .
$$

The function $x \in C([0, T], X)$ which satisfies (1) is said to be a mild solution of (2). Existence and uniqueness of a mild solution follows from the fixed point theorem; see for example [25, p. 184] or [27]. Under various additional assumptions [25], [17], [14], [11], [23] one can show that the mild solution actually satisfies (2); in particular, when $A \in \mathscr{B}(X)$, this can be easily shown. For a connection between mild solutions and weak solutions, see [2]. Local versions of the theorem can be found in [10], [25], and they lead, as in [24], to local versions of the following theorem. See also [3].

2.3. THEOREM. Assume $\mathrm{H} 1$ through $\mathrm{H} 7$. Let $E(t), t \geq 0$, be the strongly continuous semigroup whose generator is - A. Suppose $T \in(0, \infty), F:[0, T] \times X \rightarrow$ $X$ is continuous and that for some $L \in[0, \infty)$ we have that

$$
\|F(t, x)-F(t, y)\| \leq L\|x-y\| \quad \text { for } t \in[0, T], x \in X, y \in X .
$$

Choose any $y \in X$. Then, for each $n \geq 1$ there exists a unique $x_{n} \in C^{1}\left([0, T], X_{n}\right)$ such that

$$
x_{n}(0)=P_{n} y \quad \text { and } \quad x_{n}^{\prime}(t)+A_{n} x_{n}(t)=P_{n} F\left(t, Q_{n} x_{n}(t)\right) \quad \text { for } t \in[0, T] .
$$

Moreover,

$$
\lim _{n \rightarrow \infty} \sup _{0 \leq t \leq T}\left\|x(t)-Q_{n} x_{n}(t)\right\|=0,
$$

where $x \in C([0, T], X)$ satisfies

$$
x(t)=E(t) y+\int_{0}^{t} E(t-s) F(s, x(s)) d s \quad \text { for } t \in[0, T] .
$$

Proof. Existence and uniqueness of $x_{n}$ is given in Theorem 2.2, which also implies that for $t \in[0, T], n \geq 1$, we have that

$$
Q_{n} x_{n}(t)=Q_{n} E_{n}(t) P_{n} y+\int_{0}^{t} Q_{n} E_{n}(t-s) P_{n} F\left(s, Q_{n} x_{n}(s)\right) d s .
$$

This implies that for $t \in[0, T], n \geq 1$, we have that

$$
\begin{aligned}
x(t)-Q_{n} x_{n}(t)= & E(t) y-Q_{n} E_{n}(t) P_{n} y \\
& +\int_{0}^{t}\left(E(t-s)-Q_{n} E_{n}(t-s) P_{n}\right) F(s, x(s)) d s \\
& +\int_{0}^{t} Q_{n} E_{n}(t-s) P_{n}\left(F(s, x(s))-F\left(s, Q_{n} x_{n}(s)\right)\right) d s .
\end{aligned}
$$


Define

$$
\begin{gathered}
r_{n}(t)=\left\|x(t)-Q_{n} x_{n}(t)\right\|, \\
\varepsilon_{n}=\sup _{0 \leq t \leq T}\left\|E(t) y-Q_{n} E_{n}(t) P_{n} y\right\|, \quad C=p q M\left(1+e^{-a T}\right), \\
d_{n}(s)=\sup _{0 \leq t \leq T}\left\|\left(E(t)-Q_{n} E_{n}(t) P_{n}\right) F(s, x(s))\right\| \leq 2 C\|F(s, x(s))\|
\end{gathered}
$$

and note that $\lim _{n \rightarrow \infty} \varepsilon_{n}=0$ by Theorem $2.1, \lim _{n \rightarrow \infty} \int_{0}^{T} d_{n}(s) d s=0$ by Theorem 2.1 and the DCT. Since

$$
r_{n}(t) \leq \varepsilon_{n}+\int_{0}^{T} d_{n}(s) d s+L C \int_{0}^{t} r_{n}(s) d s \quad \text { for } t \in[0, T], n \geq 1,
$$

Gronwall's lemma implies that for $t \in[\dot{0}, T], n \geq 1$, we have that

$$
r_{n}(t) \leq\left(\varepsilon_{n}+\int_{0}^{T} d_{n}(s) d s\right) e^{L C T} .
$$

This completes the proof.

3. Applications to Variational Problems. In this section a linear operator $A$ will be obtained from the variational theory, and the results of Section 2 will then be applied to approximate solutions of the corresponding "parabolic" problem $\left(u^{\prime}+A u=F(\cdot, u)\right)$ and of the corresponding "wave" equation $\left(u^{\prime \prime}+A u=F\left(\cdot, u, u^{\prime}\right)\right)$. The following assumptions appear in the linear variational theory [1], [7], [8], [9], [17], [21], [24].

V1: $\mathscr{H}$ is a complex Hilbert space with inner product $(\cdot, \cdot)$ and the corresponding norm $\|\cdot\|$.

V2: $\mathscr{H}_{1}$ is a dense subspace of $\mathscr{H}$; moreover, $\mathscr{H}_{1}$ is a Hilbert space with inner product $[\cdot, \cdot]$ and norm $|\cdot|$. There exists $M_{1} \in(0, \infty)$ such that

$$
\|x\| \leq M_{1}|x| \text { for all } x \in \mathscr{H}_{1} .
$$

V3: $\mathfrak{F}: \mathscr{H}_{1} \times \mathscr{H}_{1} \rightarrow \mathrm{C}$ is a sesquilinear form, and there exist $M_{2}, M_{3}$ in $(0, \infty)$ and $a \in \mathbf{R}$ such that

$$
\begin{gathered}
|\mathfrak{F}(x, y)| \leq M_{2}|x||y| \quad \text { for } x, y \in \mathscr{H}_{1}, \\
\operatorname{Re}(\mathfrak{F}(x, x)) \geq M_{3}|x|^{2}+a\|x\|^{2} \quad \text { for } x \in \mathscr{H}_{1} .
\end{gathered}
$$

Proof of the following representation theorem (or its equivalent) can be found in many places; see, for example, [24], [17, p. 322].

3.1. THEOREM. Assume V1, V2, V3. Then, there exists a closed densely defined linear operator $A$ in $\mathscr{H}$ with the following additional properties:

(1) if $\lambda \in \mathbf{R}$ and $\lambda<a+M_{3} M_{1}^{-2}$, then $\lambda$ is in the resolvent set of $A$ and

$$
\left\|(A-\lambda)^{-1}\right\| \leq 1 /\left(a+M_{3} M_{1}^{-2}-\lambda\right) \text {; }
$$

(2) $\mathscr{D}(A) \subset \mathscr{H}_{1}, \mathscr{D}(A)$ is dense (in the $|\cdot|$ norm) in $\mathscr{H}_{1}$, and if $x \in \mathscr{H}$, $\varepsilon \in\left[0, M_{3}\right), \lambda \in\left(-\infty, a+\varepsilon M_{1}^{-2}\right]$, then

$$
\left|(A-\lambda)^{-1} x\right| \leq\|x\|\left(M_{3}-\varepsilon\right)^{-1 / 2}\left(a+M_{3} M_{1}^{-2}-\lambda\right)^{-1 / 2} ;
$$

(3) $\mathscr{F}(x, y)=(A x, y)$ for all $x \in \mathscr{D}(A), y \in \mathscr{H}_{1}$; 
(4) $M_{3}|\operatorname{Im}((A x, x))| \leq M_{2} \operatorname{Re}((A x-a x, x))$ for $x \in \mathscr{D}(A)$;

(5) $x \in \mathscr{D}(A)$ if and only if $x \in \mathscr{H}_{1}$ and there exists $z \in \mathscr{H}$ such that $\mathfrak{F}(x, y)=$ $(z, y)$ for all $y \in \mathscr{H}_{1}$

(6) if $w \in \mathscr{H}$ and $\lambda \in\left(-\infty, a+M_{3} M_{1}^{-2}\right)$, then there exists a unique $x \in \mathscr{H}_{1}$ such that $\mathfrak{F}(x, y)=(\lambda x+w, y)$ for all $y \in \mathscr{H}_{1} ;$ moreover, $x=(A-\lambda)^{-1} w$.

Observe that (1) and (4) of the above theorem imply that $-A$ is the generator of an analytic semigroup [17, p. 492] and

$$
\left\|e^{-A t}\right\| \leq \exp \left(-\left(a+M_{3} M_{1}^{-2}\right) t\right) \quad \text { for } t \geq 0 .
$$

$A$ can be considered as a generalized "elliptic" operator [21], [31]. In order to approximate solutions of "elliptic" problems, one usually makes at least the following additional assumption:

V4: Let $\mathscr{V}_{1}, \mathscr{V}_{2}, \ldots$ be finite-dimensional subspaces of $\mathscr{H}_{1}$ such that

$$
\lim _{n \rightarrow \infty} \inf _{z \in \mathscr{V}_{n}}|y-z|=0
$$

for all $y$ in a dense (in the $|\cdot|$ norm) subset of $\mathscr{H}_{1}$.

3.2. THEOREM. Assume V1, V2, V3, V4. Choose any $w \in \mathscr{H}$ and $\lambda \in$ $\left(-\infty, a+M_{3} M_{1}^{-2}\right)$. Then, for each $n \geq 1$ there exists a unique $x_{n} \in \mathscr{V}_{n}$ such that

$$
\mathfrak{F}\left(x_{n}, z\right)=\left(\lambda x_{n}+w, z\right) \text { for all } z \in \mathscr{V}_{n} .
$$

Moreover, if $x \in \mathscr{H}_{1}$ is such that $\mathfrak{F}(x, y)=(\lambda x+w, y)$ for all $y \in \mathscr{H}_{1}$, then

$$
\lim _{n \rightarrow \infty}\left|x_{n}-x\right|=0 \text {. }
$$

This is the often used result of Lax, Milgram and Cea [7, p. 104], [8, p. 327], [24]. Note that very general elliptic problems can be formulated so that the assumptions V1, V2, V3, V4 are satisfied-this includes problems in unbounded domains, very singular and degenerate problems; see, for example, [1], [6], [7], [21], [24], [26]. The following theorem shows that the convergence of approximations of solutions of the corresponding weakly nonlinear "parabolic" problem follows immediately from these assumptions and from Theorem 2.3. This result has, in effect, been obtained in [24] by a completely different proof. Many similar results have been obtained, for example [8], [9], [11], [12], [13], [16], [22], [28], under different conditions (typically, $A$ is selfadjoint with compact resolvent, or simply $A=-\Delta$ in a bounded domain with nice boundary); however, in these works the emphasis is on different topics (attractors in [11], [12], nonlinearity in [12], [13], [24], convergence rates in [13], [16], [28]) or on different types of problems [8], [9], [22].

3.3. Theorem. Assume V1, V2, V3, V4. Suppose $T \in(0, \infty), F:[0, T] \times$ $\mathscr{H} \rightarrow \mathscr{H}$ is continuous and that for some $L \in[0, \infty)$ we have that

$$
\|F(t, x)-F(t, y)\| \leq L\|x-y\| \quad \text { for } t \in[0, T], x \in \mathscr{H}, y \in \mathscr{H} .
$$

Choose any $y \in \mathscr{H}$. Then, for each $n \geq 1$ there exists a unique $u_{n} \in C^{1}\left([0, T], \mathscr{V}_{n}\right)$ such that for all $z \in \mathscr{V}_{n}$ we have $\left(u_{n}(0), z\right)=(y, z)$ and

$$
\frac{d}{d t}\left(u_{n}(t), z\right)+\mathfrak{F}\left(u_{n}(t), z\right)=\left(F\left(t, u_{n}(t)\right), z\right) \quad \text { for } t \in[0, T] .
$$


Moreover,

$$
\lim _{n \rightarrow \infty} \sup _{0 \leq t \leq T}\left\|u(t)-u_{n}(t)\right\|=0
$$

where $u \in C([0, T], \mathscr{H})$ satisfies $(A$ is as in Theorem 3.1$)$

$$
u(t)=e^{-A t} y+\int_{0}^{t} e^{-A(t-s)} F(s, u(s)) d s \quad \text { for } t \in[0, T] .
$$

Proof. Let $X_{n} \equiv \mathscr{V}_{n}$ be equipped with the norm $\|\cdot\|$ (inherent to $\mathscr{H}$ ). Let $P_{n}$ be the orthogonal (in $\mathscr{H}$ ) projections of $\mathscr{H}$ onto $X_{n}$ and let $Q_{n}$ be the identity maps from $X_{n}$ to $X \equiv \mathscr{H}$. Hence H1, H2, H3, H4, H6 are satisfied.

If we apply Theorem 3.1 where both $\mathscr{H}$ and $\mathscr{H}_{1}$ in V1, V2, V3 are replaced by $\mathscr{V}_{n}$, and if we denote the corresponding linear operator by $A_{n}$, then (1) implies

$$
\left\|\exp \left(-A_{n} t\right)\right\| \leq \exp \left(-\left(a+M_{3} M_{1}^{-2}\right) t\right) \quad \text { for } t \geq 0, n \geq 1
$$

hence $\mathrm{H} 5$ is satisfied. If $w \in X, \lambda \in\left(-\infty, a+M_{3} M_{1}^{-2}\right)$, and if $x, x_{n}$ are as in Theorem 3.2, then (6) of Theorem 3.1 implies that

$$
x_{n}=\left(A_{n}-\lambda\right)^{-1} P_{n} w=Q_{n}\left(A_{n}-\lambda\right)^{-1} P_{n} w, \quad x=(A-\lambda)^{-1} w,
$$

and hence Theorem 3.2 implies that $\mathrm{H} 7$ is satisfied.

Therefore, the assumptions $\mathrm{H} 1$ through $\mathrm{H} 7$ are satisfied and hence Theorem 2.3 and (3) of Theorem 3.1 imply the assertions of this theorem.

One has to make additional assumptions when studying the wave equation. The following assumption is, in effect, used in [31, pp. 427-430].

V5: Assume V1, V2, V3 and that for some $b \in[0, \infty)$ we have that

$$
|\operatorname{Re}(\mathfrak{F}(x, y)-\overline{\mathfrak{F}(y, x)})| \leq b\left(|x|^{2}+\|y\|^{2}\right)
$$

for all $x, y$ in a dense (in the $|\cdot|$ norm) subset of $\mathscr{H}_{1}$.

It is not hard to see that V5 implies (and is implied by)

$$
|\mathfrak{F}(x, y)-\overline{\mathfrak{F}(y, x)}| \leq 2 b|x|\|y\| \quad \text { for all } x, y \text { in } \mathscr{H}_{1} .
$$

3.4. LemMA. Assume V1, V2, V3, V5 and let $A$ be as given by Theorem 3.1. Let $X=\mathscr{H}_{1} \times \mathscr{H} ; X$ is a Hilbert space with inner product

$$
(\{x, y\},\{z, w\})=[x, z]+(y, w) .
$$

Define $B: \mathscr{D}(B) \rightarrow X$ by $\mathscr{D}(B)=\left\{\{x, y\} \in X \mid x \in \mathscr{D}(A), y \in \mathscr{H}_{1}\right\}$,

$$
B\{x, y\}=\{-y, A x\} \quad \text { for }\{x, y\} \in \mathscr{D}(B),
$$

and let $c=\left(b+|a| M_{1}\right) /\left(2 M_{3}^{1 / 2}\right), M=\left(\max \left\{1, M_{2}+|a| M_{1}^{2}\right\} / \min \left\{1, M_{3}\right\}\right)^{1 / 2}$.

Then, $B$ is a closed, densely defined linear operator in $X$; moreover, if $\lambda \in \mathbf{R}$ and $|\lambda|>c$, then $-\lambda^{2}<a+M_{3} M_{1}^{-2}, \lambda$ is in the resolvent set of $B$, and

$$
\left\|(B-\lambda)^{-m}\right\| \leq M(|\lambda|-c)^{-m} \text { for } m=1,2,3, \ldots .
$$

Furthermore, if $\{f, g\} \in X$ and $\{x, y\}=(B-\lambda)^{-1}\{f, g)$, then

$$
x=\left(A+\lambda^{2}\right)^{-1}(g-\lambda f), \quad y=-\lambda x-f .
$$

Proof. Let us first introduce a new inner product $(\cdot, \cdot)_{\text {new }}$ in $X$ by

$$
(\{x, y\},\{z, w\})_{\text {new }}=\mathfrak{F}(x, z)+\overline{\mathfrak{F}(z, x)}-2 a(x, z)+2(y, w)
$$


and observe that the corresponding new norm satisfies

$$
2 \min \left\{1, M_{3}\right\}\|\{x, y\}\|^{2} \leq\|\{x, y\}\|_{\text {new }}^{2} \leq 2 \max \left\{1, M_{2}+|a| M_{1}^{2}\right\}\|\{x, y\}\|^{2} .
$$

If $\{x, y\} \in \mathscr{D}(B)$, then

$$
\begin{gathered}
\operatorname{Re}\left((B\{x, y\},\{x, y\})_{\text {new }}\right)=\operatorname{Re}(\mathfrak{F}(x, y)-\overline{\mathfrak{F}(y, x)}+2 a(y, x)), \\
\left|\operatorname{Re}\left((B\{x, y\},\{x, y\})_{\text {new }}\right)\right| \leq 2\left(b+|a| M_{1}\right)|x|\|y\| \leq 2 c\left(M_{3}|x|^{2}+\|y\|^{2}\right)
\end{gathered}
$$

and therefore

$$
\left|\operatorname{Re}\left((B u, u)_{\text {new }}\right)\right| \leq c\|u\|_{\text {new }}^{2} \text { for } u \in \mathscr{D}(B) \text {. }
$$

If $\lambda \in \mathbf{R}$ and $|\lambda|>c$, then $-\lambda^{2}<a+M_{3} M_{1}^{-2}$ and hence, if $\{f, g\} \in X$ and

$$
x=\left(A+\lambda^{2}\right)^{-1}(g-\lambda f), \quad y=-\lambda x-f,
$$

then $\{x, y\} \in \mathscr{D}(B)$ and $(B-\lambda)\{x, y\}=\{f, g\}$. This and (1) imply

$$
\left\|(B-\lambda)^{-1}\right\|_{\text {new }} \leq(|\lambda|-c)^{-1} \text { for } \lambda \in \mathbf{R} \text { with }|\lambda|>c .
$$

This, and the equivalence of norms, implies the conclusions of the lemma.

The following theorem is new and it also follows from Theorem 2.3. An extensive discussion of the nonlinear wave equation, with many references, can be found in [11], [12]. For some other interesting results, under different assumptions, see [3], [9], [20], [30]. Standard techniques like continuation of the approximations (ODE's) or modification of the nonlinearity in a neighborhood of a known solution can be used to extend the result, see [11].

3.5. Theorem. Assume V1, V2, V3, V4, V5. Suppose also that $T \in(0, \infty)$, $F:[0, T] \times \mathscr{H}_{1} \times \mathscr{H} \rightarrow \mathscr{H}$ is continuous and that for some $L \in[0, \infty)$ we have that

$$
\begin{aligned}
& \|F(t, x, y)-F(t, z, w)\| \\
& \quad \leq L(|x-z|+\|y-w\|) \quad \text { for } t \in[0, T], x, z \in \mathscr{H}_{1}, y, w \in \mathscr{H} .
\end{aligned}
$$

Choose any $x_{0} \in \mathscr{H}_{1}, y_{0} \in \mathscr{H}$. Then, for each $n \geq 1$ there exists a unique $u_{n}$ in $C^{2}\left([0, T], \mathscr{V}_{n}\right)$ such that for all $z \in \mathscr{V}_{n}$ we have

$$
\begin{gathered}
\left(u_{n}^{\prime \prime}(t), z\right)+\mathfrak{F}\left(u_{n}(t), z\right)=\left(F\left(t, u_{n}(t), u_{n}^{\prime}(t)\right), z\right) \quad \text { for } t \in[0, T], \\
\left(u_{n}^{\prime}(0), z\right)=\left(y_{0}, z\right), \quad\left[u_{n}(0), z\right]=\left[x_{0}, z\right] .
\end{gathered}
$$

Moreover, there exists $u \in C\left([0, T], \mathscr{H}_{1}\right) \cap C^{1}([0, T], \mathscr{H})$ such that

$$
\begin{gathered}
\lim _{n \rightarrow \infty} \sup _{0 \leq t \leq T}\left(\left|u(t)-u_{n}(t)\right|+\left\|u^{\prime}(t)-u_{n}^{\prime}(t)\right\|\right)=0, \\
u(0)=x_{0}, \quad u^{\prime}(0)=y_{0}, \\
(u, z) \in C^{2}([0, T], \mathbf{C}) \quad \text { for } z \in \mathscr{H}_{1}, \\
\frac{d^{2}}{d t^{2}}(u(t), z)+\mathfrak{F}(u(t), z)=\left(F\left(t, u(t), u^{\prime}(t)\right), z\right) \quad \text { for } z \in \mathscr{H}_{1}, t \in[0, T] .
\end{gathered}
$$

Remark. The function $u$ is also a mild (for a precise statement see the proof) solution of

$$
u^{\prime \prime}(t)+A u(t)=F\left(t, u(t), u^{\prime}(t)\right), \quad u(0)=x_{0}, u^{\prime}(0)=y_{0} .
$$


Proof of Theorem 3.5. Let $X_{n}=\mathscr{V}_{n} \times \mathscr{V}_{n}: X_{n}$ is a Hilbert space with inner product

$$
(\{x, y\},\{z, w\})=[x, z]+(y, w) .
$$

Let this also be the inner product on the Hilbert space $X=\mathscr{H}_{1} \times \mathscr{H}$.

Let $P_{n}^{\prime}$ be the orthogonal (in $\mathscr{H}_{1}$ ) projection of $\mathscr{H}_{1}$ onto $\mathscr{V}_{n}$.

Let $P_{n}^{\prime \prime}$ be the orthogonal (in $\mathscr{H}$ ) projection of $\mathscr{H}$ onto $\mathscr{V}_{n}$.

Define $P_{n} \in \mathscr{B}\left(X, X_{n}\right)$ by $P_{n}\{x, y\}=\left\{P_{n}^{\prime} x, P_{n}^{\prime \prime} y\right\}$ and note that $P_{n}$ is the orthogonal projection of $X$ onto $X_{n}$.

Let $Q_{n}$ be the identity map from $X_{n}$ to $X$.

Let $B, c, M$ be as in Lemma 3.4 and note that $B$ is densely defined.

Let us replace both $\mathscr{H}_{1}$ and $\mathscr{H}$ by $\mathscr{V}_{n}$ in V1, V2, V3, V5 (the same $a, b, M_{1}, M_{2}$, $M_{3}$ will do). Denote by $A_{n}$ the corresponding operator given by Theorem 3.1 and let $B_{n}$ be the corresponding operator given in Lemma 3.4. If $m \geq 1, \lambda<-c$, then $\left\|\left(B_{n}-\lambda\right)^{-m}\right\| \leq M(-c-\lambda)^{-m}$, hence, $\left\|\exp \left(-B_{n} t\right)\right\| \leq M e^{c t}$ for $t \geq 0$. Choose $\lambda<-c,\{f, g\} \in X$; let

$$
\left\{x_{n}, y_{n}\right\}=\left(B_{n}-\lambda\right)^{-1} P_{n}\{f, g\}, \quad\{x, y\}=(B-\lambda)^{-1}\{f, g\}
$$

and note

$$
\begin{aligned}
&\left|x_{n}-x\right|=\mid\left|\left(A_{n}+\lambda^{2}\right)^{-1}\left(P_{n}^{\prime \prime} g-\lambda P_{n}^{\prime} f\right)-\left(A+\lambda^{2}\right)^{-1}(g-\lambda f)\right| \\
& \leq\left|\left(A_{n}+\lambda^{2}\right)^{-1} P_{n}^{\prime \prime} g-\left(A+\lambda^{2}\right)^{-1} g\right| \\
&+\left|\lambda \|\left(A_{n}+\lambda^{2}\right)^{-1} P_{n}^{\prime \prime} f-\left(A+\lambda^{2}\right)^{-1} f\right| \\
&+|\lambda \||\left(A_{n}+\lambda^{2}\right)^{-1}\left(P_{n}^{\prime} f-P_{n}^{\prime \prime} f\right) \mid, \\
&\left\|y_{n}-y\right\|=\left\|\lambda\left(x-x_{n}\right)+f-P_{n}^{\prime} f\right\| \leq M_{1}\left|\lambda\left(x-x_{n}\right)\right|+M_{1}\left|f-P_{n}^{\prime} f\right| .
\end{aligned}
$$

The terms (1) and (2) converge to 0 as $n \rightarrow \infty$ by Theorem 3.2 (the same argument as in the proof of Theorem 3.3). The assertion (2) of Theorem 3.1 implies that there exists $c_{1} \in(0, \infty)$ which depends only on $\lambda, a, M_{1}, M_{3}$ such that

$$
\begin{aligned}
\left|\left(A_{n}+\lambda^{2}\right)^{-1}\left(P_{n}^{\prime} f-P_{n}^{\prime \prime} f\right)\right| & \leq\left\|P_{n}^{\prime} f-P_{n}^{\prime \prime} f\right\| c_{1} \leq\left(\left\|P_{n}^{\prime} f-f\right\|+\left\|f-P_{n}^{\prime \prime} f\right\|\right) c_{1} \\
& \leq 2\left\|P_{n}^{\prime} f-f\right\| c_{1} \leq 2\left|P_{n}^{\prime} f-f\right| M_{1} c_{1} .
\end{aligned}
$$

Assumption V4 implies $\lim _{n \rightarrow \infty}\left|P_{n}^{\prime} f-f\right|=0$, and hence (3) converges to 0 as $n \rightarrow \infty$. Therefore,

$$
\lim _{n \rightarrow \infty}\left|x_{n}-x\right|=0, \quad \lim _{n \rightarrow \infty}\left\|y_{n}-y\right\|=0 .
$$

Therefore, $\mathrm{H} 1$ through $\mathrm{H} 7$ are satisfied (with $B_{n}, B$ in place of $A_{n}, A$ ). Theorem 2.3 implies that there exist unique $\left\{u_{n}, v_{n}\right\} \in C^{1}\left([0, T], X_{n}\right)$ such that

$$
\begin{aligned}
u_{n}^{\prime}(t) & -v_{n}(t)=0 \quad \text { for } t \in[0, T], \\
v_{n}^{\prime}(t)+A_{n} u_{n}(t) & =P_{n}^{\prime \prime} F\left(t, u_{n}(t), v_{n}(t)\right) \quad \text { for } t \in[0, T], \\
u_{n}(0) & =P_{n}^{\prime} x_{0}, \quad v_{n}(0)=P_{n}^{\prime \prime} y_{0} .
\end{aligned}
$$

Moreover,

$$
\lim _{n \rightarrow \infty} \sup _{0 \leq t \leq T}\left(\left|u(t)-u_{n}(t)\right|+\left\|v(t)-v_{n}(t)\right\|\right)=0
$$


where $\{u, v\} \in C([0, T], X)$ satisfies

$$
\{u(t), v(t)\}=e^{-B t}\left\{x_{0}, y_{0}\right\}+\int_{0}^{t} e^{-B(t-s)}\{0, F(s, u(s), v(s))\} d s \quad \text { for } t \in[0, T] .
$$

Since

$$
u_{n}(t)=u_{n}(0)+\int_{0}^{t} v_{n}(s) d s
$$

the limit relation (4) implies

$$
u(t)=u(0)+\int_{0}^{t} v(s) d s
$$

and therefore $u \in C^{1}([0, T], \mathscr{H})$.

To see that $u$ is also a weak solution, pick $z \in \mathscr{H}_{1}$ and note that

$$
\left(u_{n}^{\prime}(t), P_{n}^{\prime} z\right)-\left(u_{n}^{\prime}(0), P_{n}^{\prime} z\right)=\int_{0}^{t}\left(\left(F\left(s, u_{n}(s), u_{n}^{\prime}(s)\right), P_{n}^{\prime} z\right)-\mathfrak{F}\left(u_{n}(s), P_{n}^{\prime} z\right)\right) d s
$$

for $t \in[0, T], n \geq 1$. Using (4) again completes the proof.

4. Spectral Galerkin Approximations. Throughout this section it will be assumed that

$\mathrm{S} 1: \mathscr{H}$ is a complex Hilbert space with inner product $(\cdot, \cdot)$ and the corresponding norm $\|\cdot\|$.

S2: $\varphi_{n} \in \mathscr{H}$ for $n \geq 1$; let $\mathscr{V}_{n}$ denote the collection of all linear combinations of $\varphi_{1}, \ldots, \varphi_{n}$ and assume that $\bigcup_{i=1}^{\infty} \mathscr{V}_{i}$ is dense in $\mathscr{H}$.

S3: $S: \mathscr{D}(S) \equiv \bigcup_{i=1}^{\infty} \mathscr{V}_{i} \rightarrow \mathscr{H}$ is a linear operator such that for some $r \in \mathbf{R}$ and some $\gamma \in(0, \infty)$ we have that

$$
\gamma|\operatorname{Im}((S x, x))| \leq \operatorname{Re}((S x, x))+r\|x\|^{2} \text { for all } x \in \mathscr{D}(S) .
$$

It is well known [24], [26], [17, p. 325] that there exist a subspace $\mathscr{H}_{1}$ of $\mathscr{H}$, an inner product $[\cdot, \cdot]$ on $\mathscr{H}_{1}$ and a sesquilinear form $\mathfrak{F}$ on $\mathscr{H}_{1}$ such that the assumptions V1, V2, V3 of Section 2 are satisfied and that also the following holds:

(a) $\mathscr{D}(S)$ is dense (in the $|\cdot|$ norm) subspace of $\mathscr{H}_{1}$;

(b) $[x, y]=((S x, y)+(x, S y)) / 2+(1+r)(x, y)$ for $x, y$ in $\mathscr{D}(S)$;

(c) $\mathfrak{F}(x, y)=(S x, y)$ for $x, y$ in $\mathscr{D}(S)$.

The operator $A$ given by Theorem 3.1 is an extension of $S$ and is called the Friedrichs extension of $S[17$, p. 325]. Observe that (a) implies V4.

This enables us to simply replace assumptions V1, V2, V3, V4 in Theorems 3.2, $3.3,3.5$ by assumptions $\mathrm{S} 1, \mathrm{~S} 2, \mathrm{~S} 3$. While it is usually not difficult in applications to verify $\mathrm{V} 1, \mathrm{~V} 2, \mathrm{~V} 3, \mathrm{~V} 4$, it is clear that verification of $\mathrm{S} 1, \mathrm{~S} 2, \mathrm{~S} 3$ is completely elementary (with the possible exception of $\mathrm{S} 2$ ). The price one has to pay for this simplification is: $\varphi_{n} \in \mathscr{D}(S) \subset \mathscr{D}(A), \mathscr{V}_{n} \subset \mathscr{V}_{n+1}$. Thus, this approach is not suitable for the usual finite element approximations; however, it is ideal when polynomial (or other smooth functions) are used as basis functions.

4.1. TheOREM. Assume S1, S2, S3. Suppose $T \in(0, \infty), F:[0, T] \times \mathscr{H} \rightarrow \mathscr{H}$ is continuous and that for some $L \in[0, \infty)$ we have that

$$
\|F(t, x)-F(t, y)\| \leq L\|x-y\| \quad \text { for } t \in[0, T], x \in \mathscr{H}, y \in \mathscr{H} .
$$


Choose any $x_{0} \in \mathscr{H}$. Then, for each $n \geq 1$ there exists a unique $u_{n} \in C^{1}\left([0, T], \mathscr{V}_{n}\right)$ such that for all $z \in \mathscr{V}_{n}$ we have $\left(u_{n}(0), z\right)=\left(x_{0}, z\right)$ and

$$
\frac{d}{d t}\left(u_{n}(t), z\right)+\left(S u_{n}(t), z\right)=\left(F\left(t, u_{n}(t)\right), z\right) \quad \text { for } t \in[0, T] .
$$

Moreover, there exists $u \in C([0, T], \mathscr{H})$ such that

$$
\lim _{n \rightarrow \infty} \sup _{0 \leq t \leq T}\left\|u(t)-u_{n}(t)\right\|=0
$$

furthermore, this $u$ is the unique element of $C([0, T], \mathscr{H})$ which satisfies

$$
u(t)=e^{-A t} x_{0}+\int_{0}^{t} e^{-A(t-s)} F(s, u(s)) d s \quad \text { for } t \in[0, T] .
$$

This follows immediately from Theorem 3.3 and the preceding discussion. A similar result can be found in [24]. Observe how easily the assumptions can be verified. Translation of Theorem 3.2 is left to the reader; or, see [24].

The following hypothesis is obviously the appropriate modification of V5:

S4: Assume S1, S2, S3 and that there exists $b \in[0, \infty)$ such that

$$
|\operatorname{Re}((S x, y)-(x, S y))| \leq b\left(|x|^{2}+\|y\|^{2}\right) \text { for } x, y \text { in } \mathscr{D}(S) \text {. }
$$

Recall that if $x \in \mathscr{D}(S)$ then $|x|$ is evaluated as follows:

$$
|x|^{2}=\operatorname{Re}((S x, x))+(1+r)\|x\|^{2} \quad \text { for } x \in \mathscr{D}(S) .
$$

In the following theorem an adaptation of Theorem 3.5 is presented; the assumption on the nonlinearity of $F$ looks complicated on account of the fact that $F$ is defined only on $[0, T] \times \mathscr{D}(S) \times \mathscr{D}(S)$-other formulations are possible.

4.2. Theorem. Assume S1, S2, S3, S4. Suppose also that $T \in(0, \infty)$ and that $F:[0, T] \times \mathscr{D}(S) \times \mathscr{D}(S) \rightarrow \mathscr{H}$ is such that for some $L \in[0, \infty)$ we have $\|F(t, x, y)-F(t, z, w)\| \leq L(|x-z|+\|y-w\|) \quad$ for $t \in[0, T], x, y, z, w \in \mathscr{D}(S)$, and that for each $t \in[0, T], \varepsilon \in(0, \infty), \rho \in(0, \infty)$ there exists $\delta \in(0, \infty)$ such that $\|F(t, x, y)-F(s, x, y)\|<\varepsilon$ whenever $s \in[0, T],|t-s|<\delta, x, y \in \mathscr{D}(S)$, $|x|+\|y\| \leq \rho$. Choose any $x_{0} \in \mathscr{H}_{1}, y_{0} \in \mathscr{H}$.

Then, for each $n \geq 1$ there exists a unique $u_{n} \in C^{2}\left([0, T], \mathscr{V}_{n}\right)$ such that for all $z \in \mathscr{V}_{n}$ we have

$$
\begin{gathered}
\left(u_{n}^{\prime \prime}(t), z\right)+\left(S u_{n}(t), z\right)=\left(F\left(t, u_{n}(t), u_{n}^{\prime}(t)\right), z\right) \quad \text { for } t \in[0, T] \\
\left(u_{n}^{\prime}(0), z\right)=\left(y_{0}, z\right), \quad\left[u_{n}(0), z\right]=\left[x_{0}, z\right] .
\end{gathered}
$$

Moreover, there exists $u \in C\left([0, T], \mathscr{H}_{1}\right) \cap C^{1}([0, T], \mathscr{H})$ such that

$$
\begin{gathered}
\lim _{n \rightarrow \infty} \sup _{0 \leq t \leq T}\left(\left|u(t)-u_{n}(t)\right|+\left\|u^{\prime}(t)-u_{n}^{\prime}(t)\right\|\right)=0 \\
u(0)=x_{0}, \quad u^{\prime}(0)=y_{0} .
\end{gathered}
$$

Proof. The Lipschitz condition and the fact that $\mathscr{D}(S)$ is dense in both $\mathscr{H}_{1}$ and $\mathscr{H}$ allows us to define, by continuity, the extension of $F$ on $[0, T] \times \mathscr{H}_{1} \times \mathscr{H}$. One can easily see that the extension is a continuous mapping of $[0, T] \times \mathscr{H}_{1} \times \mathscr{H}$ into $\mathscr{H}$, and that it satisfies the Lipschitz condition; hence, Theorem 3.5 applies. 
Verification of the hypotheses is completely elementary, except for the following possible complications: it is required that $x_{0} \in \mathscr{H}_{1}$ and that one should be able to calculate $\left[x_{0}, z\right]$. Determining whether or not $x_{0}$ belongs to $\mathscr{H}_{1}$ can be relatively difficult. The problems disappear when $x_{0} \in \mathscr{D}(S)$ (the formula for $[\cdot, \cdot]$ is given at the beginning of this section). In particular, this happens if the original problem is formulated so that $x_{0}=0$ (thus $u_{n}(0)=0$ ). For $x_{0}$ in $\mathscr{H}_{1}$, one may calculate $\left[x_{0}, z\right]$ as follows. It can be shown $\left(\mathscr{D}(A)=\mathscr{D}\left(A^{*}\right)\right.$ when $\mathrm{S} 1, \mathrm{~S} 2, \mathrm{~S} 3, \mathrm{~S} 4$ hold $)$ that there exists $S_{1}: \mathscr{D}(S) \rightarrow \mathscr{H}$ such that

$$
(S x, y)=\left(x, S_{1} y\right) \text { for all } x, y \text { in } \mathscr{D}(S)
$$

and in applications $S_{1}$ can usually be determined by integration by parts. This implies that if $x_{0} \in \mathscr{H}_{1}, z \in \mathscr{D}(S)$, then

$$
\left[x_{0}, z\right]=\left(x_{0},(1 / 2)\left(S z+S_{1} z\right)+(1+r) z\right) .
$$

In a subsequent publication we show that, if the assumptions on $F$ are strengthened slightly, then one can allow any $x_{0} \in \mathscr{H}$, and in this case $u_{n}(0)$ can be determined simply by: $\left(u_{n}(0), z\right)=\left(x_{0}, z\right)$ for all $z \in \mathscr{V}_{n}$.

We now give an application of Theorem 4.2 to the following problem:

$$
\begin{gathered}
u_{t t}=\left(p_{1} u_{x}\right)_{x}+p_{2} u_{x}+p_{3} u+\sin \left|u_{t}\right| \quad \text { for } x \in \mathbf{R}, t \geq 0, \\
u(x, 0)=0, \quad u_{t}(x, 0)=v_{0}(x) \text { for } x \in \mathbf{R},
\end{gathered}
$$

where $v_{0} \in L^{2}(\mathbf{R})$ and $p_{i}=p_{i}(x)$ satisfy

(1) $p_{1} \in C^{1}(\mathbf{R}), p_{1}(x) \in[0, \infty)$ for $x \in \mathbf{R}, p_{1}^{\prime}$ is bounded;

(2) $p_{2}$ is a complex-valued measurable function such that for some $c<\infty$

$$
\left|p_{2}(x)\right|^{2} \leq c p_{1}(x) \text { for } x \in \mathbf{R} ;
$$

(3) $p_{3} \in L^{2}(\mathbf{R})$ is real-valued and bounded from above.

Observe that $p_{1}$ can vanish on an interval, $p_{3}$ can be singular, $p_{1}$ and $p_{2}$ may not be bounded, $v_{0}$ does not have to be smooth. The problem looks abstruse, and it is not at all clear that it makes sense. However, if one chooses the basis functions to be $\varphi_{n}(x)=x^{n-1} \exp \left(-x^{2}\right), n \geq 1$, then it is clear that the Galerkin approximations are computable. An elementary calculation will show that the assumptions of Theorem 4.2 can be satisfied, and this will give us that the Galerkin approximations converge to a generalized solution. Clearly, S1, S2 are satisfied, with $\mathscr{H}=L^{2}(\mathbf{R})$. Let $\mathscr{D}(S)$ be as given in S3 and observe that $u \in \mathscr{D}(S)$ if and only if $u(x)=q(x) \exp \left(-x^{2}\right)$ for some polynomial $q$. Define

$$
S u=-\left(p_{1} u_{x}\right)_{x}-p_{3} u \text { for } u \in \mathscr{D}(S) .
$$

Since for $u \in \mathscr{D}(S)$ we have

$$
(S u, u)=\int_{-\infty}^{\infty} p_{1}(x)\left|u_{x}(x)\right|^{2} d x-\int_{-\infty}^{\infty} p_{3}(x)|u(x)|^{2} d x \geq-r\|u\|^{2},
$$

where $r=\sup _{x} p_{3}(x)$, we see that S3 holds. Since $(S u, v)=(u, S v)$ for $u, v$ in $\mathscr{D}(S)$, we have $\mathrm{S} 4$. The mapping $F:[0, \infty) \times \mathscr{D}(S) \times \mathscr{D}(S) \rightarrow \mathscr{H}$ should be given by

$$
F(t, u, v)=p_{2} u_{x}+\sin |v|
$$


Observe that for $u, v, f, g$ in $\mathscr{D}(S)$ we have

$$
\|F(t, u, v)-F(t, f, g)\| \leq\left\|p_{2}\left(u_{x}-f_{x}\right)\right\|+\|v-g\|
$$

and since

$$
\begin{aligned}
\left\|p_{2}\left(u_{x}-f_{x}\right)\right\|^{2} & \leq c \int_{-\infty}^{\infty} p_{1}(x)\left|u_{x}(x)-f_{x}(x)\right|^{2} d x \\
& \leq c(S(u-f), u-f)+c r\|u-f\|^{2} \leq c|u-f|^{2}
\end{aligned}
$$

we see that all assumptions of Theorem 4.2 are satisfied (for any $T \in(0, \infty)$ ).

It is clear that the nonlinearity of $F$ does not have to be "local"-singular nonlinear integral operators could be added to the equation. Much worse singularities could be allowed if weighted $L^{2}(\mathbf{R})$ would be used [6]. Theorem 4.2 obviously applies also to systems.

An $L^{2}$ space is often the obvious appropriate choice for $\mathscr{H}$; however, for "nice" problems one can often choose a Sobolev space for $\mathscr{H}$, and thus approximations that converge in the Sobolev space are obtained. [5] may be helpful for verifying $\mathrm{S} 2$ in such cases.

Department of Mathematics

Michigan State University

East Lansing, Michigan 48824-1027

E-mail: 21144mmm@msu.bitnet

1. S. AGMON, "The coerciveness problem for integro-differential forms," J. Analyse Math., v. 6, 1958, pp. 183-223.

2. J. M. BALL, "Strongly continuous semigroups, weak solutions and the variation of constants formula," Proc. Amer. Math. Soc., v. 63, 1977, pp. 370-373.

3. H. T. BANKS \& K. KUNISCH, "An approximation theory for nonlinear partial differential equations with applications to identification and control," SIAM J. Control Optim., v. 20, 1982, pp. 815-849.

4. P. BRENNER \& V. THOMÉE, "On rational approximations of semigroups," SIAM J. Numer. Anal., v. 16, 1979, pp. 683-694.

5. C. CANUTO \& A. QUARTERONI, "Approximation results for orthogonal polynomials in Sobolev spaces," Math. Comp., v. 38, 1982, pp. 67-86.

6. S. N. CHOW, D. R. DUNNINGER \& M. MIKLAVČIČ, "Galerkin approximations for singular elliptic and semilinear parabolic problems," SIAM J. Numer. Anal., submitted.

7. P. G. Ciarlet, The Finite Element Method for Elliptic Problems, North-Holland, New York, 1978.

8. R. Glowinski, Numerical Methods for Nonlinear Variational Problems, Springer, New York, 1984.

9. R. GLOWINSKI, J.-L. LIONS \& R. TRÉMOlières, Numerical Analysis of Variational Inequalities, North-Holland, New York, 1981.

10. J. A. GoldSTEIN, Semigroups of Linear Operators and Applications, Oxford Univ. Press, New York, 1985.

11. J. K. HALE, Asymptotic Behavior of Dissipative Systems, Math. Surveys Monogr., vol. 25, Amer. Math. Soc., Providence, R. I., 1988.

12. J. K. HALE, X.-B. LIN \& G. RAUGEL, "Upper semicontinuity of attractors for approximation of semigroups and partial differential equations," Math. Comp., v. 50, 1988, pp. 89-123.

13. H.-P. HELFRICH, "Error estimates for semidiscrete Galerkin type approximations to semilinear evolution equations with nonsmooth initial data," Numer. Math., v. 51, 1987, pp. 559-569.

14. D. HENRY, Geometric Theory of Semilinear Parabolic Equations, Lecture Notes in Math., vol. 840, Springer-Verlag, New York, 1981.

15. R. HERSH \& T. KATO, "High-accuracy stable difference schemes for well-posed initial value problems," SIAM J. Numer. Anal., v. 16, 1979, pp. 670-682. 
16. C. JOHNSON, S. LARSSON, V. ThOMÉE \& L. B. WAHLBIN, "Error estimates for spatially discrete approximations of semilinear parabolic equations with nonsmooth initial data," Math. Comp., v. 49, 1987, pp. 331-357.

17. T. Kato, Perturbation Theory for Linear Operators, 2nd ed., Springer, New York, 1980.

18. T. KАто, "Remarks on pseudo-resolvents and infinitesimal generators of semigroups," Proc. Japan Acad., v. 35, 1959, pp. 467-468.

19. T. G. KURTZ, "Extensions of Trotter's operator semigroup approximation theorems," $J$. Funct. Anal., v. 3, 1969, pp. 354-375.

20. P. K. LAMM \& K. A. MURPHY, "Estimation of discontinuous coefficients and boundary parameters for hyperbolic systems," Quart. Appl. Math., v. 46, 1988, pp. 1-22.

21. J. L. LIONS \& E. MAGEnES, Non-Homogeneous Boundary Value Problems and Applications, Springer, New York, 1972.

22. E. Magenes, R. H. Nochetto \& C. Verdi, "Energy error estimates for a linear scheme to approximate nonlinear parabolic problems," RAIRO Modél. Math. Anal. Numér., v. 21, 1987, pp. 655-678.

23. M. MIKLAVČIČ, "Stability for semilinear parabolic equations with noninvertible linear operator," Pacific J. Math., v. 118, 1985, pp. 199-213.

24. M. MIKLAVČIČ, "Galerkin approximations of semilinear parabolic equations," preprint, 1986.

25. A. PAZY, Semigroups of Linear Operators and Applications to Partial Differential Equations, Springer, New York, 1983.

26. M. SCHECHTER, "On the invariance of the essential spectrum of an arbitrary operator II," Ricerche Mat., v. 16, 1967, pp. 3-26.

27. I. SEGAL, "Nonlinear semigroups," Ann. of Math., v. 78, 1963, pp. 339-364.

28. V. Тноме́E, Galerkin Finite Element Methods for Parabolic Problems, Lecture Notes in Math., vol. 1054, Springer, New York, 1984.

29. H. F. TROTTER, "Approximation of semi-groups of operators," Pacific J. Math., v. 8, 1958, pp. 887-919.

30. T. UshiJima, "Approximation theory for semi-groups of linear operators and its application to approximation of wave equations," Japan. J. Math., v. 1, 1975, pp. 185-224.

31. K. YosidA, Functional Analysis, Springer, New York, 1980. 